American Journal of Software Engineering and Applications
2017; 6(2): 13-17
http://www.sciencepublishinggroup.com/j/ajsea
doi: $10.11648 /$ jajsea.20170602.11
ISSN: $2327-2473$ (Print); ISSN: $2327-249 \mathrm{X}$ (Online)

\title{
Medical Image Segmentation by Active Contour Improvement
}

\author{
Abdelaziz Essadike, Elhoussaine Ouabida, Abdenbi Bouzid \\ Optronic and Information Treatment Team, Atomic, Mechanical, Photonic and Energy Laboratory, Faculty of Science, Moulay Ismail \\ University, Zitoune, Meknès, Morocco
}

Email address:

essadikeab@gmail.com (A. Essadike), contact.ouabida@gmail.com (E. Ouabida), ab2.bouzid@gmail.com (A. Bouzid)

\section{To cite this article:}

Abdelaziz Essadike, Elhoussaine Ouabida, Abdenbi Bouzid. Medical Image Segmentation by Active Contour Improvement. American Journal of Software Engineering and Applications. Vol. 6, No. 2, 2017, pp. 13-17. doi: 10.11648/j.ajsea.20170602.11

Received: March 13, 2017; Accepted: March 22, 2017; Published: April 3, 2017

\begin{abstract}
This paper introduces a new medical image segmentation approach based on active contour improvement. The boundaries in brain images are detected using an original technique of active contour improved by a Region of Interest (ROI) extraction. We compare the results of the proposed model to Chane-Vese active contour model and Caselles's et al. Geodesic active contour model. Experimental results of brain boundary localization on a national center of Oncology's database demonstrate the promising performance of this method.
\end{abstract}

Keywords: Medical Image Segmentation, Active Contours, Energy Minimization, ROI, Level Sets

\section{Introduction}

Medical images including CT (Computed Tomography) contain several anatomical structures. Accurate segmentation of one object is quite important for diagnosis and therapeutic interventional planning. Brain image segmentation methods must incorporate models that describe prior knowledge about the expected geometry and intensity characteristics of the anatomical objects of interest in the images.

The simplest image segmentation methods, such as region growing and edge detection, rely entirely on local image operators and a heuristic grouping of neighboring pixels with similar local photometric characteristics.

Active contour models have widely been used for image segmentation after they were formally introduced by Kass and Witkin [1] in 1987 as the first model. The use of level set theory has enriched the implementation of active contours with more flexibility and simplicity. The past models of active contours rely on a gradient based stopping function to stop the curve evolution.

An active contour is a curve that evolves from an initial shape to the borders of an object of interest, under the action of a force.

Active contour models can be categorized into parameterized ([1] - [5]) and non-parameterized models ([6] [17]). This classification is based on how object boundary is detected.

The active contour $C$ can be defined as a parametric curve by:

$$
\begin{aligned}
& C:[\alpha, \beta] *[0, T] \\
& (\rho, \tau) \mapsto C(\rho, \tau)=\left(\begin{array}{l}
x(\rho, \tau) \\
y(\rho, \tau)
\end{array}\right)
\end{aligned}
$$

The evolution of the contour is governed by a general form of equation:

$$
\begin{aligned}
& \frac{\partial C(\rho, \tau)}{\partial \tau}=F(\rho, \tau) \\
& C(\rho, \tau)=C_{0}(\rho)
\end{aligned}
$$

Where $C_{0}(\rho)$ is the initial contour.

The contour is deformed by following a force $\mathrm{F}$ whose direction is a priori arbitrary.

In this article we propose to use a non-parameterized active contour model to segment a skull image in order to build a 3D reconstruction of human brain. The active contour model proposed use the theory of ROI (Region of Interest) to detect the initial contour in the skull image. This initial contour minimizes the number of iterations and thus minimize the computation time but with great accuracy. 
This article is organized as follows. The previous work is reviewed in section 2 . Then the proposed model and algorithm are described in section 3. Section 4 shows the results of our experiments. Finally a brief conclusion comes in section 5 .

\section{Previous Works}

The active contours takes their origin from elastic models, but the community agrees to attribute them to the team Kass,Witkin and Terzopoulos [2], who introduced the snakes or theminimizing curves. SNAKES models take their name from their ability to deform like snakes.

Let $\Omega$ be a bounded open subset of $\mathbb{R}^{2}$, with $\partial \Omega$ itsboundary. Let $U_{0}: \Omega \rightarrow \mathbb{R}^{2}$ be a given image, and $C(s):[0,1] \rightarrow \mathbb{R}^{2}$ be a parameterized curve. This classicalmodel is inf $j_{1}(C)$ where:

$$
j_{1}(C)=\alpha \int_{0}^{1}\left|C^{\prime}(s)\right|^{2} d s+\beta \int_{0}^{1}\left|C^{\prime \prime}(s)\right|^{2} d s-\lambda \int_{0}^{1}\left|\nabla u_{0}(C(s))\right|^{2} d s
$$

Here $\alpha, \beta$ and $\lambda$ are positive parameters.

Active contours approaches can be linked to the family ofgeodesic optimization problems in spaces whose geometry isdetermined by the dynamics of the image. These techniques,called geodesic active models have been proposed by Caselleset al. [4] as:

$$
\left\{\begin{array}{l}
\frac{\partial \Phi}{\partial t}=g\left(\left|\nabla u_{0}\right|\right) \cdot|\nabla \Phi| \cdot\left(\operatorname{div}\left(\frac{\nabla \Phi}{|\nabla \Phi|}\right)+v\right), \operatorname{in}\left[0,+\infty\left[\times \mathbb{R}^{2}\right.\right. \\
\Phi(0, x, y)=\Phi_{0}(x, y), \text { in } \mathbb{R}^{2}
\end{array}\right.
$$

Where $v \geq 0$ is a constant, $\Phi$ is a Lipschitz function, represent a curve $C$ by $C=\{(x, y) / \Phi(x, y)=0\}, \Phi_{0}$ initiallevel set function,

$$
g\left(\left|\nabla u_{0}(x, y)\right|\right)=\frac{1}{1+\left|\nabla G_{\sigma}(x, y) * u_{0}(x, \mathrm{y})\right|^{p}}
$$

For, $p=2$ where $G_{\sigma} * u_{0}$, a smoother version of $u_{0}$, is the convolution of the image $u_{0}$ with the Gaussian

$$
G_{\sigma}(x, y)=\sigma^{-1 / 2} \cdot e^{\frac{-\left|x^{2}+y^{2}\right|}{4 \sigma}}
$$

The function $g(|\nabla u 0|)$ is positive in homogeneous regions, and zero at the edges.

Caselles et al. [3] proposed an new model called Geodesic Active Contour model (GAC), which is equivalent to the weighted total variation as shown by Bresson et al. [15], and it is given as: inf $J_{2}(C)$, where:

$$
\inf _{C} j_{2}(C)=2 \int_{0}^{1}\left|C^{\prime}(s)\right| \cdot g\left(\left|\nabla u_{0}(C(s))\right|\right) d s
$$

When the term $\mathrm{g}\left(\left|\nabla \mathrm{u}_{0}(\mathrm{C}(\mathrm{s}))\right|\right)$ disappears, a minimizer will be obtained. The formulation of the geodesic model by using level set method can be written as:

$$
\left\{\begin{array}{l}
\frac{\partial \Phi}{\partial t}=|\nabla \Phi| \cdot\left[\operatorname{div}\left(g(|\nabla u 0|) \cdot \frac{\nabla \Phi}{\nabla \Phi \mid}\right)+v \cdot g(|\nabla u 0|)\right], \in\left[0,+\infty\left[\times \mathbb{R}^{2}\right.\right. \\
\Phi(0, x, y)=\Phi_{0}(x, y), \in \mathbb{R}^{2}
\end{array}\right.
$$

While many segmentation methods rely heavily, in some way, on edge detection, the "Active Contours without Edges" method by Chan and Vese [6] ignores edges completely. This method is inspired by the Mumford-Shah model [18]. Chan and Vese approximate the image $\mathrm{u}_{0}$ by two regions of approximatively piecewise-constant intensities, of distinct values, object region with $\mathrm{u}_{0}^{\mathrm{i}}$, rest of the image with $\mathrm{u}_{0}^{0}$, and $\mathrm{C}_{0}$ object boundary. This object boundary is a minimizer of the following "fitting" term:

$$
\begin{aligned}
& F_{1}(C)+F_{2}(C)=\int_{\text {inside }(C)}\left|u_{0}(x, y)-c_{1}\right|^{2} d x d y \\
& +\int_{\text {outside }(C)}\left|u_{0}(x, y)-c_{2}\right|^{2} d x d y
\end{aligned}
$$

Where $C$ is any other variable curve, and the constants $C_{1}, c_{2}$, depending on $C$, are the averages of $u_{0}$ inside $\mathrm{C}$ and respectively outside $\mathrm{C}$. Chane and Vese in their model introduce the energy functional $F\left(c_{1} ; c_{2} ; C\right)$, defined by:

$$
\begin{gathered}
F\left(c_{1}, c_{2}, \mathrm{C}\right)=\mu . \text { length }(C)+v . \text { area }(\text { inside }(C)) \\
+\lambda_{1} \int_{\text {inside }(C)}\left|u_{0}(x, y)-c 1\right|^{2} d x d y \\
+\lambda_{2} \int_{\text {outside }(C)}\left|u_{0}(x, \mathrm{y})-c 2\right|^{2} d x d y
\end{gathered}
$$

Where $\mu \geq 0, v \geq 0, \lambda_{1} \lambda_{2} \succ 0$ are fixed parameters.

In almost all their numerical calculations, Chane and Vese fix these parameters as: $\lambda_{1}=\lambda_{2}=1$ and $v=0$.

\section{Description of the Model}

\subsection{Active Contour Improvement by ROI}

In our model we use the parameter $v$ different from zero, and we use the ROI extraction to calculate the area of the object.

Visual attention is not equal to all regions in the visual field but focus on a few conspicuous visual targets which are called ROI (region of interest). It exactly the same case in image processing specially in brain image database that we have. 
To define an initial contour for the active contour we use this theory of ROI by calculating the average of colons and lines to define a mask used as the initial contour:

Let $H \times L$ be the size of the CT image $u_{0}(i, j)$ of a brain.

We calculate the average on the lines to create a horizontal mask (Fig.1-b):

$$
m_{j}=\sum_{i=0}^{H} u_{0}(i, j)
$$

We calculate the average on the columns to create a vertical mask (Fig.1-c):

$$
m_{i}=\sum_{j=0}^{L} u_{0}(i, j)
$$

We calculate the product of these two masks:

$$
M=m_{j} * m_{i}
$$

Which makes the total mask of the image which is the region of interest (Fig.1.-d).

This total mask is used as an initial contour to segment the image. This initial contour minimizes the number of iterations and the computation time.

a

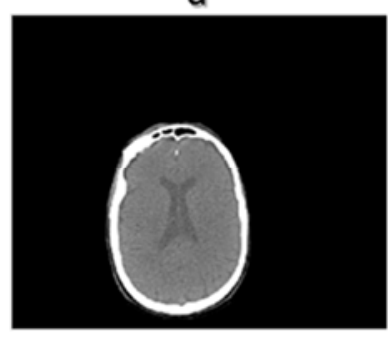

C

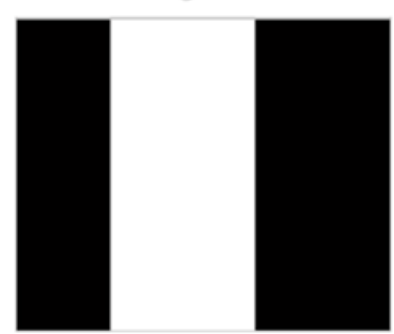

Figure 1. a) Original image, b) horizontal mask, c) vertical mask, d) total mask.

\subsection{Level Set Formulation of the Model}

For the level set formulation of this improved active contour model, we follow steps made by Chane and Vese.

By using the Heaviside function $H_{\mathcal{E}}(\mathcal{\varepsilon} \rightarrow 0)$ :

$$
H_{\mathcal{E}}(z)=\frac{1}{2} \cdot\left(1+\frac{2}{\pi} \operatorname{artan}\left(\frac{z}{\varepsilon}\right)\right)
$$

And the one-dimensional Dirac measure $\delta_{\mathcal{E}}\left(\delta_{\mathcal{E}}=H_{\mathcal{E}}^{\prime}\right)$ we express the terms in the energy, and constants $c_{1}, c_{2}$, in the following way:

$$
\begin{gathered}
F_{\mathcal{E}}\left(c_{1}, c_{2}, C\right)=\mu \int_{\Omega} \delta_{\mathcal{E}}(\Phi(x, y))|\nabla \Phi(x, y)| d x d y \\
+v \int_{\Omega} H_{\mathcal{E}}(\Phi(x, y)) d x d y+\lambda_{1} \int_{\Omega}\left|u_{0}-c_{1}\right|^{2} H_{\mathcal{E}}(\Phi(x, y)) d x d y \\
+\lambda_{2} \int_{\Omega}\left|u_{0}-c_{2}\right|^{2}\left(1-H_{\mathcal{E}}(\Phi(x, y))\right) d x d y \\
c_{1}(\Phi)=\frac{\int_{\Omega} u_{0}(x, y) \cdot H_{\mathcal{E}}(\Phi(x, y)) d x d y}{\int_{\Omega} H_{\mathcal{E}}(\Phi(x, y)) d x d y}
\end{gathered}
$$

$$
c_{2}(\Phi)=\frac{\int_{\Omega} u_{0}(x, y) \cdot\left(1-H_{\mathcal{E}}(\Phi(x, y))\right) d x d y}{\int_{\Omega}\left(1-H_{\mathcal{E}}(\Phi(x, y))\right) d x d y}
$$

\subsection{Numerical Approximation of the Model}

For the numerical approximation of this model, we use a finite difference implicit scheme similar to Chane and Vese approximation by following Rudin et al. [19] for the discretization of the divergence operator in Lipschitz function expression, by taking: $h$ the space step, $\Delta t$ the time step,

$\left(x_{i}, y_{j}\right)=(i h, j h)$ the grid points, $\lambda_{1}=\lambda_{2}=1$ and $v=1$

$$
\begin{aligned}
& \Phi^{n}=\Phi\left(\mathrm{n} \Delta t, \mathrm{x}_{i}, y_{j}\right) \\
& \Delta_{-}^{x} \Phi_{i, j}=\Phi_{i, j}-\Phi_{i-1, j} \\
& \Delta_{+}^{x} \Phi_{i, j}=\Phi_{i+1, j}-\Phi_{i, j} \\
& \Delta_{-}^{y} \Phi_{i, j}=\Phi_{i, j}-\Phi_{i, j-1} \\
& \Delta_{+}^{y} \Phi_{i, j}=\Phi_{i, j+1}-\Phi_{i, j}
\end{aligned}
$$

to get the following linear system solved by an iterative method:

$$
\frac{\Phi_{i, j}^{n+1}-\Phi_{i, j}^{n}}{\Delta t}=\delta_{h}\left(\Phi_{i, j}^{n}\right)\left[\begin{array}{c}
\frac{\mu}{h^{2}} \Delta_{-}^{x} \cdot\left(\frac{\Delta_{+}^{x} \Phi_{i, j}^{n+1}}{\frac{\left(\Delta_{+}^{x} \Phi_{i, j}^{n+1}\right)^{2}}{h^{2}}+\frac{\left(\Phi_{i, j+1}^{n}-\Phi_{i, j-1}^{n}\right)^{2}}{2 h^{2}}}\right) \\
+\frac{\mu}{h^{2}} \Delta_{-\cdot}^{y} \cdot\left(\frac{\Delta_{+}^{y} \Phi_{i, j}^{n+1}}{\frac{\left(\Delta_{+}^{y} \Phi_{i, j}^{n+1}\right)^{2}}{h^{2}}}+\left(\Phi_{i+1, j}^{n}-\Phi_{i-1, j}^{n}\right)^{2}\right. \\
-v-\lambda_{1}\left(u_{i, j}-c_{1}\left(\Phi^{n}\right)\right)^{2}+\lambda_{2}\left(u_{i, j}-c_{2}\left(\Phi^{n}\right)\right)^{2}
\end{array}\right]
$$

Therefore, as it was also pointed out by Chane and Vese, their model is a particular case of the minimal partition 
problem, for which the existence of minimizers of the energy $F\left(c_{1}, c_{2}, C\right)$ has been proved in [18], for that, and in order to compute the associated Euler-Lagrange equation, we use the regularized functional $F\left(c_{1} ; c_{2} ; C\right)$ :

$$
\begin{aligned}
& \frac{\partial \Phi}{\partial t}=\delta_{\varepsilon}(\Phi)\left[\mu \operatorname{div}\left(\frac{\nabla \Phi}{|\nabla \Phi|}-v-\lambda_{1}\left(u-c_{1}\right)^{2}+\lambda_{2}\left(u-c_{2}\right)^{2}\right]=0\right. \\
& \Phi(0, x, y)=\Phi_{0}(x, y) \in \Omega
\end{aligned}
$$

\section{Experiments}

\subsection{Images Database Used in Experiments}

This database used in our experiment is from Meknes Oncology Centre contains a total of 41 brain images. All the brain images are 8 bit color PNG files (Fig. 2).
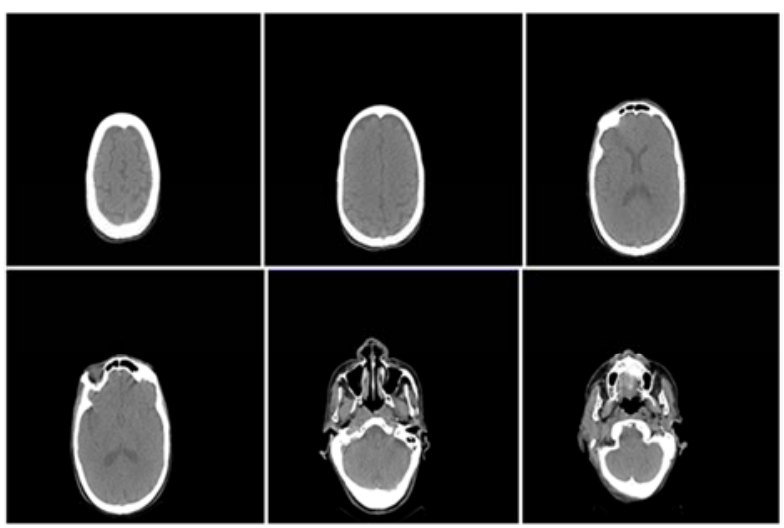

Figure 2. Examples of brain images from the database used in experiments.

\subsection{Experimental Results}

In this section, we present experiments to evaluate the performance of our proposed model. We will present the iterations number, the elapsed time by seconds and segmented image percentages performed on a 1,4 GHz AMD E1-1200 with 4Go of RAM. For each iteration from 1 to 1000 , and for each brain images, we calculate the elapsed time and the segmented image percentage with our model, and then we compare it with the Chane-Vese [6] and the Caselles et al. [3] models where we use the same initial contour used in Chane and Vese experiments. All of the experiments were performed in Matlab version 2015a (Mathworks, Natick, CA).
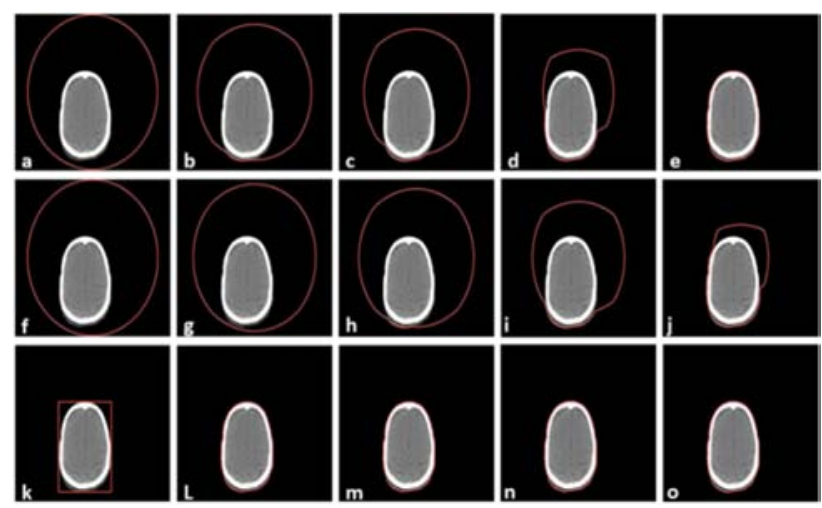

Figure 3. Segmentation of the brain image database.

a) are the original image and initial contour, b)-c)-d)-e) are segmentation results of Chane-Vese model for 100, 200, 500 and 1000 iterations respectively. f) are the original image and initial contour, g)-h)-i)-j) are segmentation results of Caselleset al. model for 100, 200, 500 and 1000 iterations respectively. k) are the original image and initial contour detected by the ROI extraction, L)-m)-n)-o) are segmentation results of our model for 100,500 and 1000 iterations respectively.

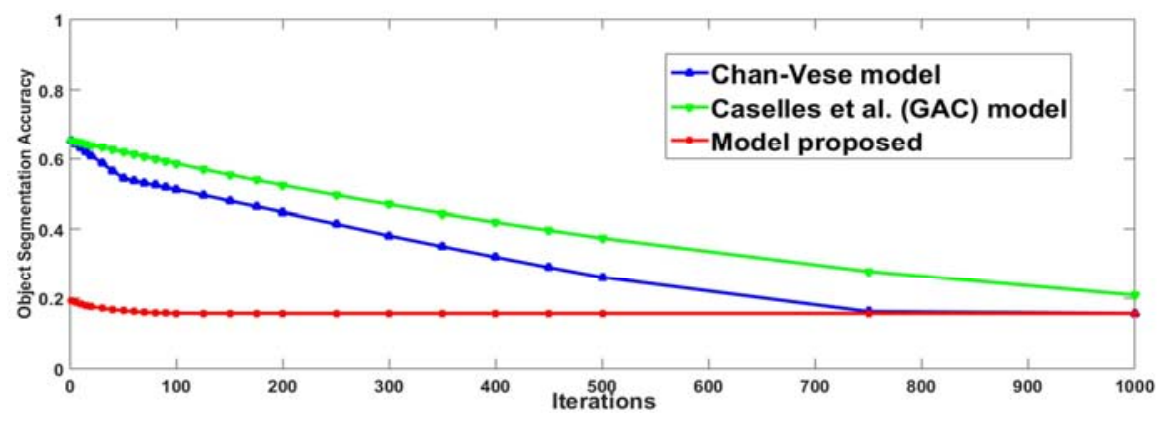

Figure 4. Comparisons of segmented image percentage (accuracy) along with iterations.

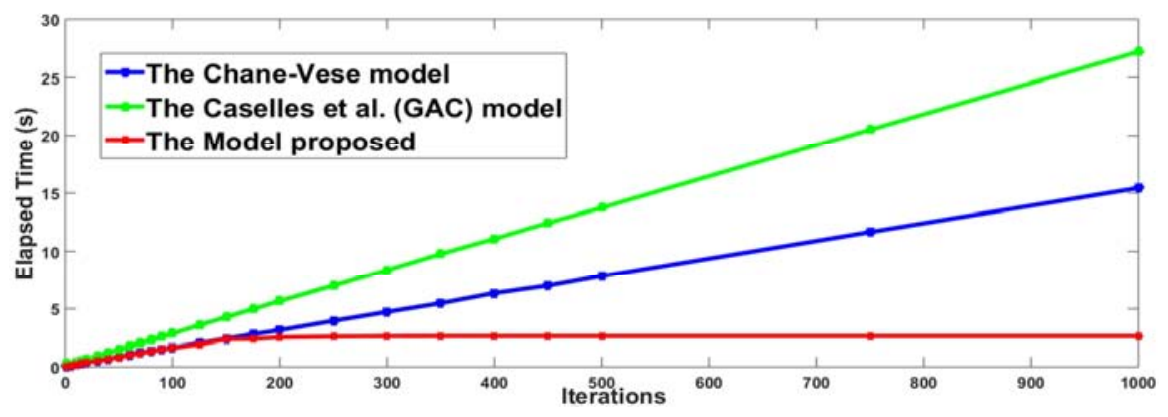

Figure 5. Comparisons of the elapsed time along with iterations. 
125 iterations for a time of 1.945559 seconds (Fig. 5) and for a pixels segmented percentage of $15.68 \%$ from a $537 \times 637$ pixels image. Unlike our model, the curves of Fig. 3 show that the other two models have problems in the accuracy of brain segmentation with time of 15.432051 seconds and 27.213132 seconds for the Chane-Vese model and Caselles et al. model respectively after 1000 iterations (Fig. 3-e-j).

Comparing the results in Fig. 4, we notice that the Chance-Vese model is faster than Caselles et al. model and slower than our model, but it reaches its performance after 1000 iterations for 15.432051 seconds as elapsed time.

Once the brain images are segmented, an edge detector filter is used to present sections of the brain volume (Fig. 6).
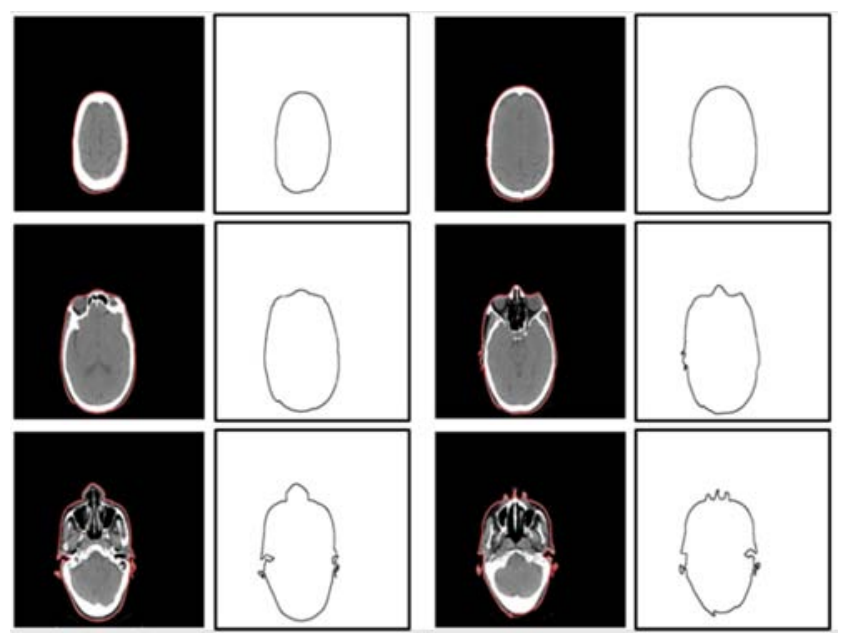

Figure 6. Sections show the segmented brain series.

\section{Conclusion}

In this paper, we have proposed a new improved active contour model with a Region Of interest (ROI) extraction. ROI is extracted by calculating the average of colons and lines to define a mask used as the initial contour. This model has been applied to the Meknes Oncology Centre databases. Based on the results presented above, we can conclude that the proposed method is encouraging and outperforms most of the existing active contours models in terms of segmentation accuracy and iterations number and the elapsed time.

\section{References}

[1] M. Kass, A. Witkin and D. Terzopoulos, "Snake: active contours model,” Int. J. Comput. Vis., vol. 1, pp. 1167-1186, 1991.

[2] L. Cohen, "On active contour models and balloons," Comput. Graph. Image Process., vol. 53, pp. 211-218, 1991.

[3] V. Caselles, R. Kimmel and G. Sapiro, "Geodesic active contours,” Int. J. Comput. Vis., vol. 22, no. 1, pp. 61-79, 1997.
[4] C. Xu and J. Prince, "Snakes, shapes, and gradient vector flow," IEEE Trans. Image Process., vol. 10, pp. 359-369, 1998.

[5] C. Xu and J. Prince, "Generalized gradient vector flow external forces for active contours," Signal Process., vol. 71, no. 2, pp. 131-139, 1998.

[6] T. Chan and L. Vese, "Active contours without edges," IEEE Trans. Image Process., vol. 10, no. 2, pp. 266-277, 2001.

[7] C. Li, C. Kao, J. Gore and Z. Ding, "Minimization of region-scalable fitting energy for image segmentation," IEEE Trans. Image Process., vol. 17, pp. 1940-1949, 2008.

[8] L. Wang, L. He, A. Mishra and C. Li, "Active contours driven by local gaussian distribution fitting energy," Signal Process., vol. 89, no. 12, pp. 2435-2447, 2009.

[9] X. Bresson and T. Chan, "Non-local Unsupervised Variational Image Segmentation Model," UCLA CAM Report, pp. 08-67, 2008 .

[10] K. Ni, X. Bresson, T. Chan and S. Esedoglu, "Local histogram based segmentation using the Wasserstein distance," Int. J. Comput. Vis., pp. 97-111, 2009.

[11] K. Zhang, H. Song and L. Zhang, "Active contours driven by local image fitting energy," Pattern Recognit., vol. 43, no. 4, pp. 1199-1206, 2010.

[12] T. Brox and D. Cremers, "On the statistical interpretation of the piecewise smooth Mumford-Shah functional," Scale Space Var. Methods Comput. Vis., pp. 203-213, 2007.

[13] T. Chan, S. Esedoglu and M. Nikolova, "Algorithms for finding global minimizers of image segmentation and denoising models," SIAM J. Appl. Math., vol. 66, pp. 1632-1648, 2006.

[14] T. Goldstein, X. Bresson and S. Osher, "Geometric applications of the split Bregman method: segmentation and surface reconstruction,” J. Sci. Comput., vol. 45, no. 1, pp. 272-293, 2010 .

[15] X. Bresson, S. Esedoglu, P. Vandergheynst, J. Thiran and S. Osher, "Fast global minimization of the active contour/snake model," J. Math. Imaging Vis., vol. 28, pp. 151-167, 2007.

[16] M. Jung, G. Peyr and L. Cohen, "Non-local active contours," SIAM J. Imaging Sci., vol. 5, no. 3, pp. 1022-1054, 2012.

[17] P. Yan, W. Zhang, B. Turkbey, P. Choyke and X. Li, "Global structure constrained local shape prior estimation for medical image segmentation," Comput. Vis. Image Understand., vol. 117, pp. 1017-1026, 2013.

[18] D. Mumford and J. Shah, "Optimal approximations of piecewise smooth functions and associated variational problems," Commun. Pure Appl. Math., vol. 42, pp. 577-685, 1989.

[19] L. Rudin, S. Osher and E. Fatemi, "Nonlinear total variation based noise removal algorithms," Phys. D, vol. 60, pp. 259-268, 1992. 